\title{
Challenges in elucidating cholangiocarcinoma etiology
}

\author{
Sarah S. Jackson ${ }^{1}$, Andrea A. Florio ${ }^{1}$, Katherine A. McGlynn ${ }^{1}$, Jessica L. Petrick ${ }^{2}$ \\ ${ }^{1}$ Division of Cancer Epidemiology and Genetics, National Cancer Institute, NIH, DHHS, Bethesda, MD, USA; ${ }^{2}$ Slone Epidemiology Center, \\ Boston University, Boston, MA, USA \\ Correspondence to: Jessica L. Petrick, PhD, MPH. 72 East Concord Street, L-7, Boston, MA 02118, USA. Email: jpetrick@bu.edu. \\ Provenance and Peer Review: This article was commissioned by the editorial office, Hepatobiliary Surgery and Nutrition. The article did not undergo \\ external peer review. \\ Comment on: Clements O, Eliahoo J, Kim JU. Risk factors for intrahepatic and extrahepatic cholangiocarcinoma: A systematic review and meta- \\ analysis. J Hepatol 2020;72:95-103.
}

Submitted Jan 23, 2020. Accepted for publication Feb 06, 2020.

doi: 10.21037/hbsn.2020.02.03

View this article at: http://dx.doi.org/10.21037/hbsn.2020.02.03

Cholangiocarcinomas (CCAs) are rare tumors that originate from cholangiocytes in the bile ducts and are classified as intra- or extrahepatic (ICC or ECC). ICCs account for 10 $12 \%$ of liver cancers, while ECCs account for approximately one-third of biliary tract cancers (1). Incidence rates of CCA are geographically variable, with the highest rates in Asia. However, even in the highest incidence country (South Korea) the rates are only 2.8 and 2.2 per 100,000 personyears for ICC and ECC, respectively (2). Incidence rates of both ICC and ECC have been increasing in most countries globally, which potentially indicates a changing etiology (2).

There are few established risk factors for CCA, including chronic conditions-primary sclerosing cholangitis and Caroli's disease-and, in Asian countries, liver flukes and hepatolithiasis. However, the rarity of CCA makes elucidating the etiology of these tumors challenging. Compounding the challenges in studying CCA etiology is the evolving ICD coding over time, which has resulted in misclassification of perihilar or Klatskin tumors, which are ECCs arising proximal to the cystic duct (3).

In response to these issues, we have conducted large, pooled analyses of prospective cohort studies-the Liver Cancer Pooling Project (LCPP) and the Biliary Tract Cancers Pooling Project (BiTCaPP). These pooling projects have allowed us to examine harmonized risk factors for ICC and ECC, with a focus on Western populations. In the BiTCaPP, we identified cigarette smoking and obesity, but not alcohol consumption, as etiologic factors that increase ECC risk $(4,5)$. In the LCPP, we identified excess alcohol consumption, cigarette smoking, diabetes and obesity as ICC risk factors $(6,7)$. Further, we conducted a systematic review and meta-analysis and reported that obesity and diabetes were associated with a $50 \%$ increased ICC risk (7). We only included studies with pre-diagnostic assessment of obesity and diabetes (i.e., cohort and nested case-control), as studies that assessed exposure at or after time of cancer diagnosis are susceptible to reverse causation, as ICC patients often present with cachexia.

In the January issue of the fournal of Hepatology, Clements et al. conducted a systematic review and meta-analysis of risk factors for ICC and ECC (8). To conduct this study, the authors searched a singular source (MEDLINE) to identify published articles. They reported that bile duct cysts and stones, chronic hepatitis $\mathrm{B}$ and $\mathrm{C}$ viral infections, and inflammatory bowel disease increased risk of ICC and ECC 2- to 35-fold. More modest increased risk of ICC and ECC were noted for alcohol, smoking, and diabetes, while no associations were reported for hypertension or obesity (8).

The objective of systematic reviews and meta-analyses are to rigorously review and synthesize the entire body of scientific studies - that is, to determine if there is replication in the published literature. The study by Clements et al. is the largest and most comprehensive meta-analysis to dateexamining 13 potential risk factors in 25 studies of ICC and ECC (8). However, producing an averaged effect estimate across all studies may come at the expense of identifying and reporting heterogeneity in study-specific estimates, which can illuminate why certain studies did not replicate prior findings. The authors present the individual forest and funnel plots, which show minimal publication bias and 
homogeneous results for most exposures. However, the study-specific estimates are not consistent for smoking, alcohol, hypertension, and obesity.

The cautious reader should apply the same scrutiny to a systematic review and meta-analysis that they would to any other study, carefully considering potential sources of bias (9). In Clements et al., study selection is a potential concern. First, the authors only included case-control studies, which is not justified or explained. However, this includes a mixture of case-control and nested casecontrol study designs. Nested case-control studies, by definition, are nested within larger cohort studies, with exposure nearly always assessed pre-diagnostically. While in case-control studies, exposure is typically recalled or assessed at diagnosis. Therefore, the requirement for all included studies to be case-control in design did not alleviate heterogeneity in exposure assessment timing. This suggests that reverse causation could have contributed to the Clements et al. study findings. A second potential source of bias is inclusion of duplicate study populations. For example, in the smoking-ICC meta-analysis, three overlapping Surveillance, Epidemiology, and End Results Program-Medicare studies were included. If the duplicate studies are removed, the meta-analytic results are no longer significant. Finally, discrepancies in study-specific exposure assessment could contribute to bias and heterogeneous results; for instance, alcohol exposure was defined by both a range of grams per day and alcoholic liver disease (8).

A primary aim of the Clements et al. study was to compare the etiologies of ICC and ECC. The systematic review excluded studies prior to 1990, when the second version of the International Classification of Diseases for Oncology (ICD-O) was published and provided a unique histology code for perihilar tumors (8162/3). However, ICD-O-2 cross-referenced this morphology code to the intrahepatic bile duct (C22.1), while ICD-O-3 crossreferenced to both the intra- and extrahepatic bile ducts (C24.0). Clements et al. states that perihilar tumors account for $50-60 \%$ of all CCAs, estimates that come from single US institution reports (8). In US cancer registries, perihilar tumors only account for $5 \%$ of CCA cases (1). However, a recent study suggested that not all cancer registrars recognize that perihilar is synonymous with Klatskin (10). This is highly problematic, as many epidemiologic studies rely on cancer registries and ICD codes to classify cases. Thus, even in the few studies that reclassify the perihilar tumors as ECC there could be bias in outcome ascertainment. The newest release of ICD-O-3.2, recommended for use starting in 2020, explicitly defines perihilar and Klatskin as synonymous and cross-references these tumors to the extrahepatic bile duct only.

In sum, the study by Clements et al. underscores the challenges faced in elucidating CCA etiologylow incidence, potential reverse causation, and outcome misclassification. Further research is necessary to determine etiologic factors contributing to the increasing rates of CCA, with particular focus on CCA subtypes-anatomic and molecular-which have differing pathobiologies. Additionally, novel factors require investigation, including circulating biomarkers and the microbiome. The combination of molecular studies and revised cancer registry coding will build on current epidemiologic observations and further elucidate the etiology of CCA.

\section{Acknowledgments}

Funding: None.

\section{Footnote}

Conflicts of Interest: All authors have completed the ICMJE uniform disclosure form (available at http://dx.doi. org/10.21037/hbsn.2020.02.03). The authors have no conflicts of interest to declare.

Ethical Statement: The authors are accountable for all aspects of the work in ensuring that questions related to the accuracy or integrity of any part of the work are appropriately investigated and resolved.

Open Access Statement: This is an Open Access article distributed in accordance with the Creative Commons Attribution-NonCommercial-NoDerivs 4.0 International License (CC BY-NC-ND 4.0), which permits the noncommercial replication and distribution of the article with the strict proviso that no changes or edits are made and the original work is properly cited (including links to both the formal publication through the relevant DOI and the license). See: https://creativecommons.org/licenses/by-nc$\mathrm{nd} / 4.0 \%$.

\section{References}

1. Van Dyke AL, Shiels MS, Jones GS, et al. Biliary tract cancer incidence and trends in the United States by demographic group, 1999-2013. Cancer 2019;125:1489-98. 
2. Florio AA, Ferlay J, Znaor A, et al. Global incidence and trends in intra- and extrahepatic cholangiocarcinoma from 1993 to 2012. Cancer 2020. [In press].

3. Welzel TM, McGlynn KA, Hsing AW, et al. Impact of classification of hilar cholangiocarcinomas (Klatskin tumors) on the incidence of intra- and extrahepatic cholangiocarcinoma in the United States. J Natl Cancer Inst 2006;98:873-5.

4. McGee EE, Jackson SS, Petrick JL, et al. Smoking, Alcohol, and Biliary Tract Cancer Risk: A Pooling Project of 26 Prospective Studies. J Natl Cancer Inst 2019;111:1263-78.

5. Jackson SS, Van Dyke AL, Zhu B, et al. Anthropometric Risk Factors for Cancers of the Biliary Tract in the Biliary Tract Cancers Pooling Project. Cancer Res 2019;79:3973-82.

6. Petrick JL, Campbell PT, Koshiol J, et al. Tobacco, alcohol use and risk of hepatocellular carcinoma and intrahepatic

Cite this article as: Jackson SS, Florio AA, McGlynn KA, Petrick JL. Challenges in elucidating cholangiocarcinoma etiology. HepatoBiliary Surg Nutr 2020;9(4):537-539. doi: 10.21037/hbsn.2020.02.03 cholangiocarcinoma: The Liver Cancer Pooling Project. Br J Cancer 2018;118:1005-12.

7. Petrick JL, Thistle JE, Zeleniuch-Jacquotte A, et al. Body Mass Index, Diabetes and Intrahepatic Cholangiocarcinoma Risk: The Liver Cancer Pooling Project and Meta-analysis. Am J Gastroenterol 2018;113:1494-505.

8. Clements O, Eliahoo J, Kim JU, et al. Risk factors for intrahepatic and extrahepatic cholangiocarcinoma: A systematic review and meta-analysis. J Hepatol 2020;72:95-103.

9. Moher D, Liberati A, Tetzlaff J, et al. Preferred reporting items for systematic reviews and meta-analyses: the PRISMA statement. PLoS Med 2009;6:e1000097.

10. Khan SA, Emadossadaty S, Ladep NG, et al. Rising trends in cholangiocarcinoma: is the ICD classification system misleading us? J Hepatol 2012;56:848-54. 